\title{
Resumption of treatment with penicillamine after proteinuria
}

\author{
HILARY HILL, ALAN HILL, AND A. M. DAVISON \\ From the Oxford Regional Rheumatic Diseases Research Centre, Stoke Mandeville Hospital, Aylesbury, \\ Bucks, and the Department of Renal Medicine, St James's University Hospital, Leeds
}

SUMMARY Penicillamine has been successfully reintroduced and continued for a minimum of 13 months in 5 patients who developed proteinuria during the first course of the drug. The daily maintenance dose during the second course was $150-250 \mathrm{mg}$ taken midway between 2 meals. Proteinuria did not recur; no significant excretion of fibrin degradation products occurred; complement, urea, creatinine, and serum albumin remained within normal limits. Urine microscopy showed no abnormality.

Proteinuria is a major reason for abandoning treatment with penicillamine in patients with rheumatoid arthritis. In a personal series (Hill, 1977) 16 of 69 patients $(23 \%)$ treated with a daily maintenance dose of $750 \mathrm{mg}$ developed proteinuria exceeding $2 \mathrm{~g} / 24$ hours during the first 12 months of treatment and penicillamine was consequently withdrawn. In most of these 16 patients the disease subsequently became only slightly more active, but in a few deterioration was so severe that the patients and their families pressed strongly for resumption of treatment. Jaffe (1977-8) reported that reintroduction of penicillamine, starting with a daily dose of $250 \mathrm{mg}$, was usually followed by a return of proteinuria at about the same time and at about the same cumulative dose as on the first occasion. The object of this study was to determine whether cautious reintroduction of penicillamine could be accomplished without a return of proteinuria, and whether the use of very much smaller doses would be clinically effective.

\section{Materials and methods}

\section{ADMISSION CRITERIA}

The reintroduction of penicillamine was considered only for patients who had had a good clinical and laboratory response to the drug during the first course, whose disease was again persistently active, and who requested a further trial of penicillamine. Further conditions of entry were that the urine had

Accepted for publication 25 May 1978

Correspondence to Dr Hilary Hill, Stoke Mandeville Hospital, Mandeville Road, Aylesbury, Bucks HP21 8AL become free from protein after penicillamine was withdrawn and had remained so for at least 3 months; serum urea, creatinine, and albumin had to be within normal limits; and no red cells or casts were present on urine microscopy. Five patients fulfilled these criteria. Four of these were normotensive, the fifth had hypertension which antedated the first use of penicillamine and had not subsequently increased. There was thus no evidence of nephropathy in these 5 patients. The salient features of the first course of treatment are summarised in Table 1.

\section{DOSE}

The initial dose of penicillamine was $50 \mathrm{mg}$, increased by monthly increments of $50 \mathrm{mg}$ to a maintenance dose of $150 \mathrm{mg}$ daily. The dose was held at $150 \mathrm{mg} /$ day for 4 months and thereafter increased by further increments of $50 \mathrm{mg}$ at intervals of 3 months if the disease remained persistently active. In an attempt to standardise treatment and to ensure maximum absorption of the $50 \mathrm{mg}$ tablets patients were instructed to take their daily dose of penicillamine midway between 2 meals, so that at least $1 \frac{1}{2}$ hours elapsed after the end of one meal before penicillamine was taken and at least a further $1 \frac{1}{2}$ hours elapsed before the next meal. During this 3-hour period only water was allowed. Initially penicillamine was given in 2 divided doses, but patients who had completed 6 months of treatment were given the option of taking the drug in a single dose at a time best suited to their individual convenience. Some of these patients who woke early opted for early morning as the most convenient time, postponing breakfast if necessary. 
Table 1 First course of penicillamine

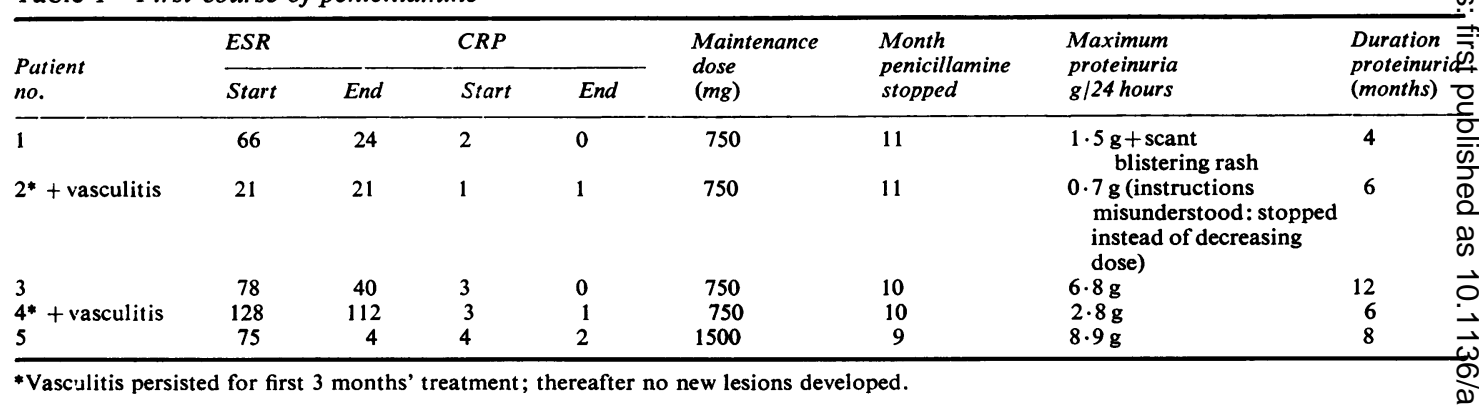

\section{MONITORING}

All patients were seen monthly at hospital by the same physician. In addition to routine mandatory haematological and urine checks measurements of complement, $\mathrm{C}$-reactive protein (CRP), urea, creatinine, and serum albumin were done at each visit, and urine was sent each week for determination of fibrin degradation products (FDP) by the tanned red cell haemagglutination inhibition immunoassay (Clarkson et al., 1970).

Clinical assessment of disease activity was based on the duration of morning stiffness, examination of affected joints, and counting the number of joints which were free from pain on a full range of nonweight bearing active movement (Hill et al., 1973). This last measure embraced 16 limb joints (metacarpophalangeal, wrist, elbow, shoulder, hip, knee, ankle, metatarsophalangeal-the mcp and mtp joints on each side being scored as single joints).

\section{Results}

No patient developed proteinuria during the first year of treatment, and urine microscopy showed no abnormality. Similarly there was no significant change in urea, creatinine, or serum albumin. Complement remained within normal limits. There was no significant FDP excretion during treatment. Blood pressures remained at pretrial levels.
The final maintenance daily dose of penicillamine was $150 \mathrm{mg}$ in 3 patients, $200 \mathrm{mg}$ in one and 250 in the fifth. Four patients reported clinical improve ment by the third month of treatment, but cone sistent objective improvement was not apparent until about 6 months had elapsed. In all 5 patients the erythrocyte sedimentation rate (ESR) fell an the number of pain-free joints increased. CRP disappeared in 3 patients and became less strongl 10 positive in 2 . Morning stiffness was virtually absent throughout treatment in 1 patient, decreased subo stantially in duration in 3 patients, but doubled is the fifth patient. These changes are shown io Table 2.

Comparison between the effect of a maintenance dose of 750 or $1500 \mathrm{mg}$ of penicillamine in the first course and 150-250 $\mathrm{mg}$ in the second can be made only in respect of ESR and CRP as the number of pain-free joints, and the duration of morning stiffness. was not recorded regularly during the first coursey Nevertheless, comparable changes in ESR an $\bar{\Phi}$ CRP during both courses suggest that the coms paratively small dose of penicillamine used in the second course did reduce disease activity.

Four of the 5 patients continue with penicillamine the maximum duration of treatment amounting te 24 months. Penicillamine was withdrawn in the fifth patient after 13 months when she developed. widespread pruritic skin lesions characteristic of

Table 2 Penicillamine reintroduced: first 12 months' results

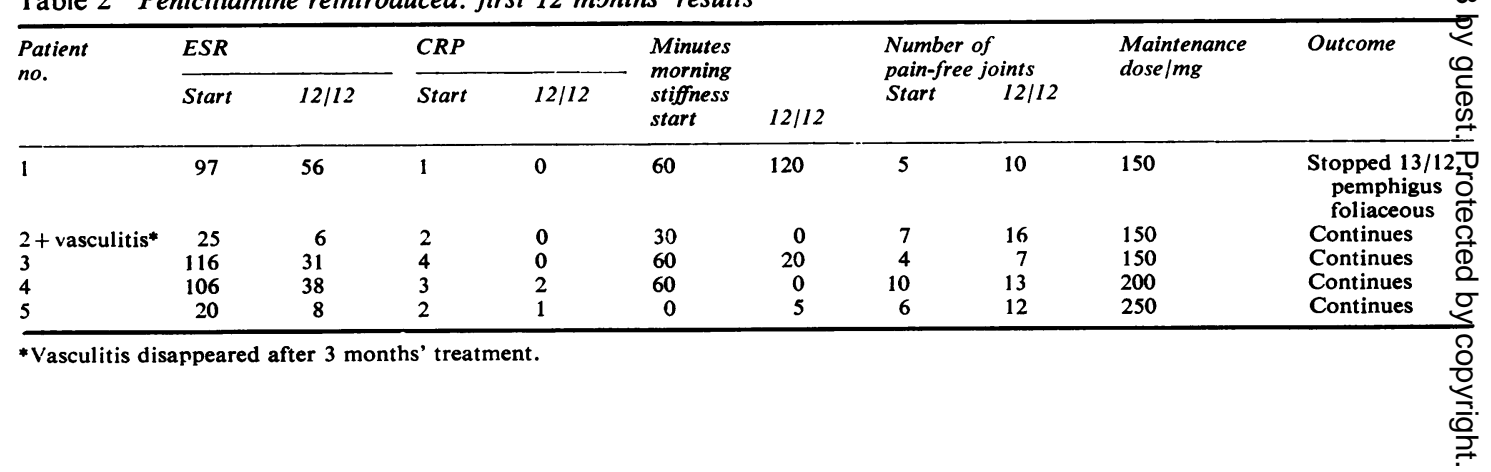


pemphigus foliaceus-another adverse effect of penicillamine (Marsden et al., 1976). In retrospect the 2 tiny blisters which accompanied the development of proteinuria during the first course of treatment in this patient were probably early lesions of pemphigus foliaceous; the lack of pruritus and the brief duration of the rash which occurred at that time may have been due to the prompt withdrawal of penicillamine.

\section{Discussion}

The object of this study was to see if penicillamine could be reintroduced without inducing proteinuria if a very low dose were used. This object has been achieved, all patients completing a minimum of 13 months of treatment, the urine remaining free from protein throughout. Proteinuria at 'normal' doses of $750 \mathrm{mg}$ or more of penicillamine occurs most often during the second 6 months of treatment. All 5 patients have passed this high risk time without recurrence of proteinuria. Proteinuria, though rare after 12 months of treatment (Hill, 1977), may occur at wide ranges of dose and length of treatment (Davison et al., 1977). Urinary FDP excretion correlates with intraglomerular fibrin deposition (Davison et al., 1973) and this relates to the severity of the glomerular inflammation and may be a more sensitive indicator of glomerular disease. The lack of any significant urinary FDP excretion during this study therefore indirectly indicates the lack of any penicillamine-induced nephropathy.

Jaffe (1977-8) found that proteinuria occurred at the same time and after the same cumulative dose if penicillamine was reintroduced at higher dose levels. In contrast, in the small group of patients in the present study reintroduction of penicillamine in a very small dose was not followed by recurrence of proteinuria, and all have now passed the time in treatment at which proteinuria occurred in the original course. However, the results do not run counter to those of Jaffe in respect of recurrence of proteinuria at the same cumulative dose level as in the first course, since none of our patients has yet had a total dose of penicillamine in the second course approaching that reached in the first course when proteinuria appeared. Even the patient who has been taking $200 \mathrm{mg} /$ day for a total of 24 months has so far reached only just over half the total dose of penicillamine which she took during the first course. Clearly the study must continue until the 'critical' cumulative dose has been reached and passed.

The decision to specify that the drug be taken between meals and with water only was prompted by the observation of Lyle et al. (1977) that the absorption of penicillamine is decreased if a single $300 \mathrm{mg}$ tablet of ferrous sulphate is taken with the drug. It is therefore conceivable that other substances including food, tea (which contains manganese), and medicines may effect the absorption of penicillamine. Thus when penicillamine is taken with meals the amount absorbed could in theory vary very widely from day to day in the same patient and between patients.

As regards efficacy, in this group of patients all have shown improvement over the 13 months of treatment. Improvement has been present in objective measures as well as in a personal sense of wellbeing, but the latter must be construed with caution as possibly due to personal conviction based on a good response during the first course that all would be well when penicillamine was restarted. It was noteworthy that objective improvement was evident only after 6 months of treatment, and without controls it is impossible to exclude natural remission rather than a specific response to penicillamine. Much larger numbers and a double blind trial can alone elucidate this. On the credit side these 5 patients have again been able to tolerate penicillamine, have not had a recurrence of proteinuria, and have apparently benefited clinically.

The support of the Yorkshire Kidney Research Fund is gratefully acknowledged.

\section{References}

Clarkson, A. R., Morton, J. B., and Cash, J. D. (1970). Urinary fibrin/fibrinogen degradation products after renal homotransplantation. Lancet, 2, 1220-1223.

Davison, A. M., Thomson, D., MacDonald, M. K., Rae, J. K., Uttley, W. S., and Clarkson, A. R. (1973). Identification of intrarenal fibrin deposition. Journal of Clinical Pathology, 26, 102-112.

Davison, A. M., Day, A. T., Golding, J. R., and Thomson, D. (1977). Proceedings of the Royal Society of Medicine, 70, Supplement 3, 109-113.

Hill, H. F. H. (1977). Treatment of rheumatoid arthritis with penicillamine. Seminars in Arthritis and Rheumatism, 6, 361-388.

Hill, H. F. H., Hill, Alan G. S., Mowat, A. G., Ansell, B. M., Matthews, J. A., Seifert, M. H., Gumpel, J. M., and Christie, G. A. (1973). Multicentre double-blind crossover trial comparing naproxen and aspirin in rheumatoid arthritis. Scandinavian Journal of Rheumatology, Supplement 2, 176-181.

Jaffe, I. A. (1977-8). D-Penicillamine. Bulletin on Rheumatic Diseases, 28, 948-952.

Lyle, W. H., Pearcey, D. F., and Hui, M. (1977). Inhibition of penicillamine-induced cupruresis by oral iron. Proceedings of the Royal Society of Medicine, 70, Supplement $3,48-49$.

Marsden, R. A., Ryan, T. J., Vanhegan, R. I., Walshe, M., Hill, H., and Mowat, A. G. (1976). Pemphigus foliaceous induced by penicillamine. British Medical Journal, 2, 1423-1424. 